\title{
Tecnura
}

\section{Análisis comparativo de la carbonización de cuesco de palma de aceite en reactores de lecho fijo}

\section{Comparative analysis of the carbonization of oil palm kernel shells in fixed bed reactors}

\author{
David Francisco Flórez Ramos¹, Jimmy Barco Burgos², Sonia Lucía Rincón Prat ${ }^{3}$
}

Fecha de recepción: 1 de abril de 2016

Fecha de aceptación: 15 de mayo de 2016

Cómo citar: Flórez Ramos, D. F., Barco Burgos, J., \& Rincón Prat, S. L. (2016). Análisis comparativo de la carbonización de cuesco de palma de aceite en reactores de lecho fijo. Revista Tecnura, 20(49), 45-58. doi: 10.14483/ udistrital.jour.tecnura.2016.3.a03

\section{RESUMEN}

Contexto: La producción de aceite crudo de palma se ha incrementado en un $60 \%$ en el país en los últimos 5 años. Este incremento va acompañado de la producción de cada vez mayores cantidades de residuos generados en el proceso de extracción. En este trabajo se evalúa el proceso de carbonización de cuesco de palma de aceite en diferentes reactores con miras a su aprovechamiento como fuente de energía renovable.

Método: La carbonización se realiza en un montaje con retorta a escala laboratorio y en dos reactores de lecho fijo a escala semi-piloto. Se comparan los rendimientos másicos y las características fisicoquímicas de los productos utilizando la misma tasa de calentamiento y aumentando el tamaño del reactor y se estudia la influencia de la velocidad de calentamiento para la retorta.

Resultados: Un aumento en la tasa de calentamiento de $2,5 \mathrm{~K} / \mathrm{min}$ a $7,0 \mathrm{~K} / \mathrm{min}$ ocasiona un $10 \%$ de disminución de carbonizado y un aumento de 1,5 $\%$ y $20 \%$ de gas y productos líquidos respectivamente. Al aumentar el tamaño del reactor, aumenta el rendimiento de carbonizado y de gas en un $6 \%$ y $7 \%$ respectivamente y disminuyen los productos líquidos en un $3 \%$.

Conclusiones: $\mathrm{Al}$ aumentar el tamaño del reactor se favorecen los efectos de transferencia de masa y calor y las reacciones secundarias. Estos efectos junto con el uso de una tasa de calentamiento baja, conllevan a mayores rendimientos del carbonizado y a mayor rendimiento gaseoso con mayor poder calorífico. Palabras clave: Carbonización, Cuesco de palma de aceite, Reactor de lecho fijo, Escalamiento.

\section{ABSTRACT}

Background: Raw palm oil production has increased $60 \%$ in the last 5 years in the country. This increase is accompanied by the production of a larger amount of residues generated during the extraction process. In this work, the carbonization of oil palm shells is assessed in different reactors in order to determine their use as a renewable energy source.

Method: Oil palm shells carbonization is carried out in a laboratory scale retort set-up and two semi-pilot scale fixed bed reactors. Mass yields and

1 Ingeniero Químico, estudiante de la maestría en Ingeniería Química, integrante del Grupo de Investigación en Biomasa y Optimización de Procesos Térmicos de la Universidad Nacional de Colombia. Bogotá, Colombia. Contacto: dafflorezra@unal.edu.co

2 Ingeniero Mecánico, magíster en Ingeniería Mecánica, integrante del Grupo de Investigación en Biomasa y Optimización de Procesos Térmicos de la Universidad Nacional de Colombia. Bogotá, Colombia. Contacto: jbarcobu@unal.edu.co

3 Ingeniera Mecánica, doctora en Ingeniería, directora del Grupo de Investigación en Biomasa y Optimización de Procesos Térmicos de la Universidad Nacional de Colombia. Bogotá, Colombia. Contacto: slrinconp@unal.edu.co 
physiochemical properties of products, are compared by increasing the size of the reactor at the same heating rate and the influence of the heating rate is studied in the retort set-up.

Results: An increase in the heating rate from 2,5 K/ min to $7,0 \mathrm{~K} / \mathrm{min}$ promotes a $10 \%$ of reduction of char and an increase of $1,5 \%$ and $20 \%$ of gas and liquid products respectively. When increasing the size of the reactor, the char and gas yields increase in $6 \%$ and $7 \%$ respectively and liquid products decrease in $3 \%$.

Conclusions: By increasing the reactor size, mass and heat transport phenomena and secondary reactions are promoted. This phenomena along with a reduction in heating rate generates larger char yields and larger gaseous yields with higher calorific value. Key words: Carbonization, Oil Palm Shells, Fixed bed reactor, Scaling.

\section{INTRODUCCIÓN}

La biomasa es entendida como toda la materia que se puede derivar de manera directa o indirecta de la fotosíntesis de las plantas, y puede encontrarse en forma vegetal o animal. Las fuentes de biomasa para uso energético pueden ser plantaciones energéticas, residuos forestales, desechos agrícolas, desechos industriales y desechos urbanos (Campero, 2010). Cuando los enlaces entre las moléculas adyacentes de carbono, hidrógeno y oxígeno se rompen por digestión, combustión o descomposición, estas sustancias liberan su energía química almacenada (McKendry, 2002). La biomasa contribuye en cerca del $10 \%$ de los combustibles como fuente de energía primaria a nivel mundial, siendo en promedio del $5 \%$ en los países industrializados y cerca del $30 \%$ en los países en vía de desarroIlo (Escalante, Orduz, Zapata, Cardona y Duarte; 2008; Faaji, 2006). Desde 1971 hasta 2011 aumentó la demanda energética en Colombia en un 125 \%, con una disminución notable de carbón mineral y aceite como fuentes de suministro y aumento en el suministro energético mediante biocombustibles, gas natural e hidroeléctricas (IEA, 2011a). En el mundo, el incremento de la demanda energética en el mismo periodo fue de alrededor de $140 \%$, para la biomasa el aumento fue del $50 \%$ siendo la mayor participación la correspondiente a la madera (IEA, 2011b). La biomasa puede mezclarse con carbón mineral o combustibles sólidos convencionales para reducir el impacato ambiental, sin afectar el desempeño de los hornos durante la combustión (González, García y Talero, 2014).

Los residuos agroindustriales comprenden una alternativa viable de aprovechamiento energético mediante procesos de transformación termoquímica. En las últimas dos décadas el cultivo de palma de aceite en Colombia ha crecido aceleradamente, pasando de 111.380 ha en 1990 a 403.684 ha en 2010. Esto se debe a la creciente demanda de grasas y aceites extraídos del fruto, que tienen múltiples usos, entre los que se destaca la producción de biodiesel (Fedepalma, 2013).

El cuesco constituye un residuo importante en los cultivos de palma de aceite; su adecuado uso y disposición presenta en la actualidad problemas importantes en este sector de la agroindustria en Colombia. Este material constituye entre 5 y $7 \%$ del peso total del racimo de fruta fresca (RFF), es de consistencia dura y en su forma natural posee estructura porosa. Para 2015 la producción mundial de cuesco de palma de aceite fue de 14.800 kt; en Colombia la producción fue de 260 kt, con un potencial energético de 2677,44 TJ/año (Núñez, 2012). El cuesco se ha usado parcialmente en combustión en calderas, ya que presenta el inconveniente de que su ceniza obstruye tuberías y ductos debido al alto contenido de sodio y potasio en forma de óxidos que contiene (Abnisa, Wan Daud y Sahu, 2011). La deformación de la ceniza para cuescos inicia en promedio a temperaturas de $800{ }^{\circ} \mathrm{C}$; para cascarillas, en cambio, la deformación empieza hacia los $1450{ }^{\circ} \mathrm{C}$, y para carbones, 
hacia los $1200{ }^{\circ} \mathrm{C}$ (Vassilev, Baxter, Andersen y Vassileva, 2013). El cuesco de palma, por su apariencia y dureza, puede ser considerado un pellet natural de material lignocelulósico, cuya densidad a granel en forma bruta y compacta, varía en el rango de 500 a $600 \mathrm{~kg} \mathrm{~m}^{-3}$ y 600 a $740 \mathrm{~kg} \mathrm{~m}^{-3}$, respectivamente (Asadullah, Ab Rasid, Sharifah y Azdarpour, 2013).

La pirólisis es la degradación térmica de la biomasa en ausencia de oxígeno. Este proceso genera productos líquidos, sólidos y gaseosos, cuya distribución y características dependen altamente de los parámetros de operación, como el tipo de biomasa, la temperatura máxima alcanzada, la tasa de calentamiento y el tiempo de permanencia de los productos en el reactor (Chen y Lin, 2016). El material sólido se puede emplear como combustible o materia prima para la producción de carbón activado; el producto líquido, Ilamado también bioaceite, se puede emplear como combustible rico en hidrocarburos; y el gas, como generador de energía mecánica o térmica, o para sintetizar productos químicos (Gómez, Klose y Rincón, 2008a). El producto líquido está formado en un 28,7 \% por aromáticos conjugados con olefinas, y en un $37 \%$ por compuestos aromáticos con radicales $\mathrm{CH}, \mathrm{CH}_{2}$ y $\mathrm{CH}_{3}$ (Ugur y Sevgi, 2015; Zhongking, Dengyu, $\mathrm{Gu}$, Bao y Qisheng, 2015). Para ser empleado como materia prima para producción de naftas y diésel necesita pasar por hidrogenación, y para producción de gasolina de alto octanaje requiere pasar por craqueo catalítico con zeolitas. En los procesos anteriores se disminuye el contenido de oxígeno y se remueven álcalis (McKendry, 2001).

La velocidad de calentamiento durante la pirólisis cumple un papel muy importante debido a que se relaciona con el tiempo de residencia de la fase volátil en el lecho de biomasa y, por tanto, con los rendimientos másicos producidos. Altas velocidades favorecen los productos fluidos y bajas velocidades el producto carbonizado (Firdaus, Uemura, Osman y Yuzup, 2015; Seneca, 2007; Basu, 2010). Entre tasas de calentamiento moderadas (10-20 K/ min), se han observado cambios significativos en el rendimiento sólido (> 5 \%) (Seon-Jin, Su-Hwa y Joo-Sik, 2010). La pirólisis puede ser Ilevada a cabo en atmósfera inerte o con concentraciones bajas de oxígeno y dióxido de carbono, aunque la eficiencia energética del material sólido tiende a disminuir con un agente externo (Uemura, Saadon, Osman y Mansor, 2015). Durante la pirólisis se descompone la hemicelulosa entre 160 y 360 ${ }^{\circ} \mathrm{C}$; la celulosa entre 240 y $400{ }^{\circ} \mathrm{C}$ y la lignina entre 500 y $600{ }^{\circ} \mathrm{C}$ (Ninduangdee, Kuprianov, Young Cha y Kaewrath, 2015).

Los experimentos en laboratorio o planta piloto permiten predecir el comportamiento y la factibilidad técnica que presentará una planta industrial en condiciones similares de operación. La planta piloto es el camino más seguro hacia la certeza de invertir en una modificación de un proceso productivo, ya que genera nuevos criterios y conocimientos para avanzar en optimización, alcance, seguridad y rentabilidad (Pedroza y Anaya, 2008).

La presente investigación tiene como objetivo realizar un análisis comparativo de la operación de pirólisis de cuesco de palma en tres reactores de lecho fijo de distintos tamaños. Los tres reactores corresponden a una retorta, un reactor de lecho fijo horizontal y un reactor de lecho fijo vertical.

\section{METODOLOGÍA}

Se llevan a cabo cuatro pruebas experimentales de pirólisis de cuesco de palma de aceite en tres montajes en operación en lote. Las condiciones experimentales de las pruebas se observan en la tabla 1.

Para todas las pruebas se utiliza cuesco de palma con tamaño de partícula entre 0,5 y $3 \mathrm{~mm}$ el cual se logra empleando un molino de cuchillas marca Retsch SM 100 neu. Las tasas de calentamiento utilizadas corresponden a los valores obtenidos en los reactores de lecho fijo a nivel de kilogramos de biomasa (prueba 2 y prueba 4), ya que en estos no es posible realizar un control de este parámetro como valor de entrada sino que el perfil de calentamiento es el resultado de la temperatura final establecida, la masa de materia prima y 
Tabla 1. Condiciones experimentales de las pruebas de pirólisis realizadas

\begin{tabular}{clccccc}
\hline $\begin{array}{c}\text { Número } \\
\text { de } \\
\text { prueba }\end{array}$ & Montaje & $\begin{array}{c}\text { Volumen del } \\
\text { reactor } \\
(\mathbf{m l})\end{array}$ & $\begin{array}{c}\text { Masa inicial } \\
\text { de la muestra } \\
\text { seca } \mathbf{( g )}\end{array}$ & $\begin{array}{c}\text { Tasa de } \\
\text { calentamiento } \\
(\mathbf{K} / \mathbf{m i n})\end{array}$ & $\begin{array}{c}\text { Temperatura } \\
\text { máxima } \\
\text { alcanzada }\left({ }^{\circ} \mathbf{C}\right)\end{array}$ & $\begin{array}{c}\text { Tiempo de } \\
\text { calentamiento } \\
\text { total }(\mathbf{m i n})\end{array}$ \\
\hline 1 & Retorta & 163,3 & 45,39 & 2,5 & 550 & 200 \\
\hline 2 & $\begin{array}{l}\text { Reactor de lecho fijo } \\
\text { vertical }\end{array}$ & 38200,0 & 11892,22 & 2,5 & 550 & 200 \\
\hline 3 & Retorta & 163,3 & 81,39 & 7,0 & 590 & 120 \\
\hline 4 & $\begin{array}{l}\text { Reactor de lecho fijo } \\
\text { horizontal }\end{array}$ & 20106,0 & 4820,11 & 7,0 & 590 & 120 \\
\hline
\end{tabular}

Fuente: elaboración propia.

el flujo de gas de arrastre utilizados, así como, de la inercia propia de cada reactor. La retorta tiene mejor capacidad de control de temperatura por lo que se reproducen los perfiles de calentamiento previamente determinados en los reactores. A continuación se presenta una descripción de los montajes empleados y el procedimiento experimental desarrollado para la realización de las pruebas de pirólisis en cada uno.

\section{Pirólisis en retorta}

El montaje experimental utilizado para llevar a cabo la pirólisis del cuesco de palma de aceite en retorta se presenta en la figura 1 . Este se basa en la norma ISO 647 "Determinación de rendimientos de alquitranes, agua, gas y carbonizado por destilación a baja temperatura".

En este montaje se llevan a cabo las pruebas experimentales cuyas condiciones corresponden a las pruebas 1 y 3 de la tabla 1 . El material a procesar se ubica en la retorta metálica (A). El calentamiento se realiza por medio de un mechero Bunsen de acuerdo con el perfil establecido. Los productos volátiles y gaseosos generados pasan a un Erlenmeyer (B) que se encuentra sumergido en un baño de agua con hielo causando la condensación de los alquitranes y el agua generados. Luego el gas es conducido por un filtro (C) y es recogido en un depósito de vidrio con agua a un nivel

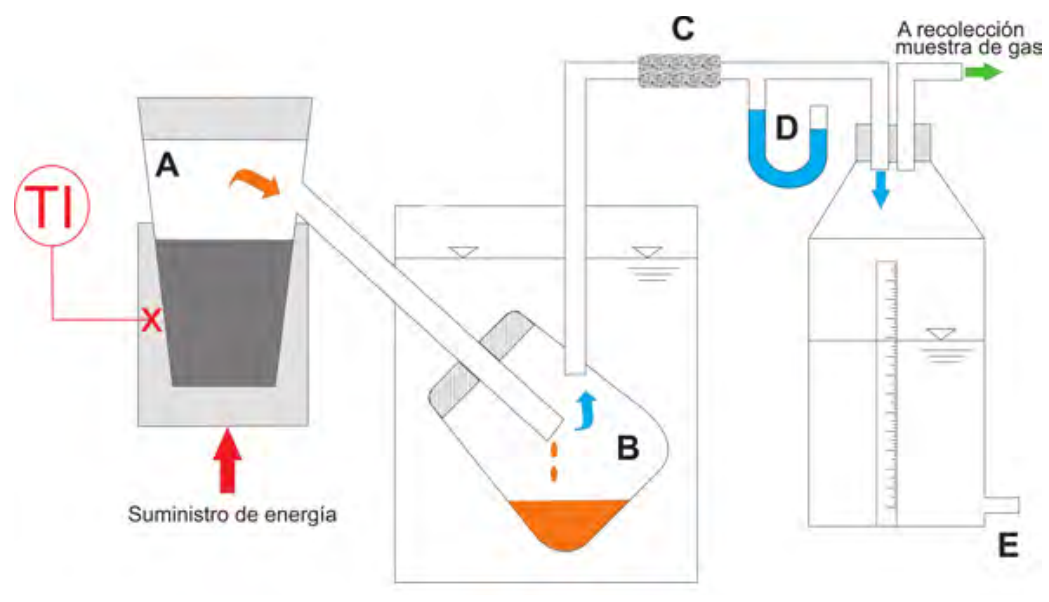

Figura 1. Montaje de retorta para pirólisis de biomasa. A. Retorta metálica, B. Erlenmeyer, C. Filtro D. tubo en U. E. Depósito con agua a nivel graduado.

Fuente: elaboración propia. 
graduado (E). La recolección se logra regulando la presión observada en un tubo en $U(\mathrm{D})$, en el interior del depósito, mediante la evacuación coordinada de agua. Después de terminar el proceso se deja enfriar el sistema hasta temperatura ambiente y se determinan la masa de carbonizado residual, la masa del residuo líquido en el Erlenmeyer, la masa de las secciones de vidrio posteriores a este último para determinar el alquitrán adherido en las paredes y el volumen final desplazado de gas. Para calcular el volumen real de gas se utiliza un factor de corrección adicionado a la lectura en el depósito de almacenamiento de vidrio. Este factor corresponde al cambio en el volumen de material sólido en la retorta, sumado al volumen de condensado, teniendo en cuenta la temperatura alcanzada durante la operación.

Para registrar las temperaturas se usa un DataLogger acoplado a un termopar tipo K. Durante el experimento, se toman cada 5 minutos lecturas del nivel de agua en el depósito de gas.

\section{Pirólisis en reactor de lecho fijo vertical}

El montaje experimental se representa en la figura 2 (a). En este montaje se lleva a cabo la prueba 2, cuyas condiciones operacionales se observan en la tabla 1. El reactor es alimentado por una tolva que contiene el material a procesar (A). Como la operación se realiza en lotes, se alimenta la biomasa, luego se retira el sistema de alimentación y se sella el equipo. El lecho es cilíndrico con una longitud de 99,5 cm y un diámetro de $16 \mathrm{~cm}$. Para el calentamiento, se emplean dos quemadores a gas marca Joannes Finterm modelo JM 3 de 11,9 a 37,7 kW de potencia en extremos opuestos del equipo. Se ubican termopares a 57, 86, 137 y $185 \mathrm{~cm}$ de la tapa superior. En cada posición axial se ubican dos termopares: uno junto a la pared del reactor y el otro a la mitad del radio del mismo, de acuerdo con la figura 2 (b). El perfil de temperaturas se registra mediante una tarjeta de adquisición de datos Agilent 34970A. La temperatura media del reactor

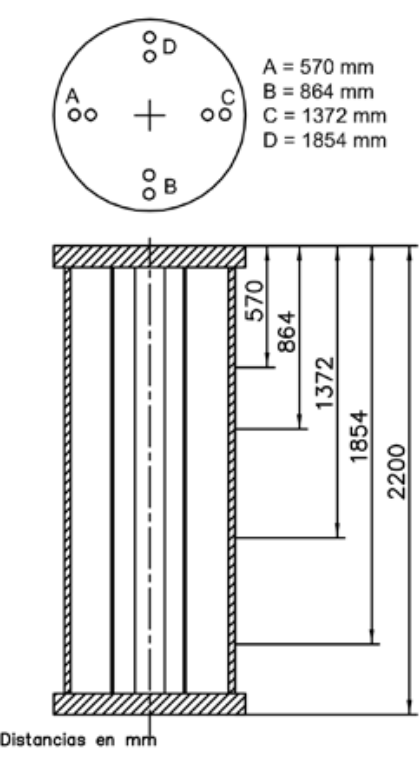

(b)

Figura 2. (a) Montaje del reactor de lecho fijo vertical para pirólisis biomasa. A. Sistema de alimentación.

B. Reactor cilíndrico. F. Intercambiadores de calor. G. Filtros de aserrín para limpieza de gases. H. Bomba de vacío. I. Contador de gas. J. Chimenea. (b) Distribución de los termopares en el reactor de lecho fijo vertical.

Fuente: elaboración propia. 
de lecho fijo vertical corresponde al promedio entre las medidas tomadas de los termoelementos B y los termoelementos $C$, ya que son estos los que miden la temperatura de la muestra.

A medida que se calienta la muestra los productos volátiles y gaseosos salen del reactor (B) succionados por una bomba de vacío $(\mathrm{H})$. Entre el reactor y la bomba de vacío se ubican dos intercambiadores de calor de tubos concéntricos $(F)$ en donde se condensan los alquitranes y el agua y dos filtros de aserrín $(\mathrm{G})$ donde se separan los alquitranes que permanecen en el gas después de la condensación. Después de la bomba, los gases son medidos por medio de un contador de gas (I). Luego, la mezcla de gases es quemada y dirigida hacia una chimenea (J). Por último se deja enfriar el equipo a temperatura ambiente y se determina la masa de carbonizado residual, la masa de residuo líquido condensado de los intercambiadores y las secciones de manguera posteriores a estos para determinar el alquitrán adherido en las paredes. En este montaje experimental se realiza la evacuación continua del gas producido, por lo que no es posible recolectar una muestra de la cantidad total de gas producida que permita determinar su composición final.

Durante el experimento se regula un flujo de nitrógeno de entrada en $5 \mathrm{I} \mathrm{min}^{-1}$, como gas de arrastre inerte, se regula un flujo de $2 \mathrm{I} \mathrm{min}^{-1}$ a la entrada de los frascos recolectores de alquitranes y 0,25 I min $^{-1}$ para el depósito de almacenamiento del gas producido y recolección de muestra. Se toma el registro del volumen indicado en el contador de gas ubicado antes de la chimenea del reactor, cada 5 minutos (I). Todos los flujos se reportan a una temperatura de $20{ }^{\circ} \mathrm{C}$ y 734,7 mbar.

\section{Pirólisis en reactor de lecho fijo horizontal}

El montaje experimental utilizado se representa en la figura 3(a) En este montaje se lleva a cabo la prueba 4, cuyas condiciones operacionales se observan en la tabla 1 . El reactor es alimentado manualmente y posteriormente sellado. Como el reactor está ubicado de manera horizontal, el lecho formado por la materia prima es semicilíndrico con una longitud de $64 \mathrm{~cm}$ y ocupa el $70 \%$ de la sección transversal circular de diámetro 16 cm. Para el calentamiento, se emplean dos series de resistencias eléctricas metálicas, situadas en los lados opuestos de la cámara de aislamiento del reactor, controlando el calentamiento a la tasa de $4,8 \mathrm{~K} / \mathrm{min}$. Se ubican termopares a 4, 30, 63,5, y $94 \mathrm{~cm}$ de la tapa de entrada del gas de arrastre y en cada posición axial se ubican tres termoelementos a 2,5, 4,7 y $7 \mathrm{~cm}$ del centro del reactor, de acuerdo con la figura 3 (b). El perfil de temperaturas se registra mediante una tarjeta de adquisición de datos Agilent 34970A, conectada a los diferentes termoelementos. La temperatura media del reactor corresponde al promedio de las medidas reportadas por los termoelementos a $30 \mathrm{~cm}$ y a $94 \mathrm{~cm}$, en donde hacen contacto con el lecho de biomasa.

El sistema de adecuación, limpieza, evacuación y muestreo del gas es el mismo empleado para el reactor de lecho fijo vertical al que se le incorporó la posibilidad de recolectar una muestra de gas. Para esto en el ducto que comunica el reactor con los intercambiadores ( $F$ ) se conecta un ducto que desvía una pequeña fracción del gas hacia un sistema de toma de muestras para posterior análisis. Este sistema cuenta con dos recipientes metálicos en serie en cada uno de los cuales se ubican tres botellas de vidrio. El primer recipiente está regulado a 35 ${ }^{\circ} \mathrm{C}$ y el segundo a $15{ }^{\circ} \mathrm{C}$. La función de este sistema es condensar los volátiles contenidos en la fracción del flujo de gas que circula (C). El gas es recolectado en un depósito de vidrio con agua a un nivel graduado $(\mathrm{E})$, regulando la presión en el interior mediante la evacuación coordinada de agua con la presión manométrica vista en el tubo en $U$ (D).

\section{Caracterización de materia prima y productos}

- Materia prima y carbonizado: Se realiza análisis próximo, determinando el contenido de humedad, contenido de ceniza y contenido de materia volátil, de acuerdo con las normas 


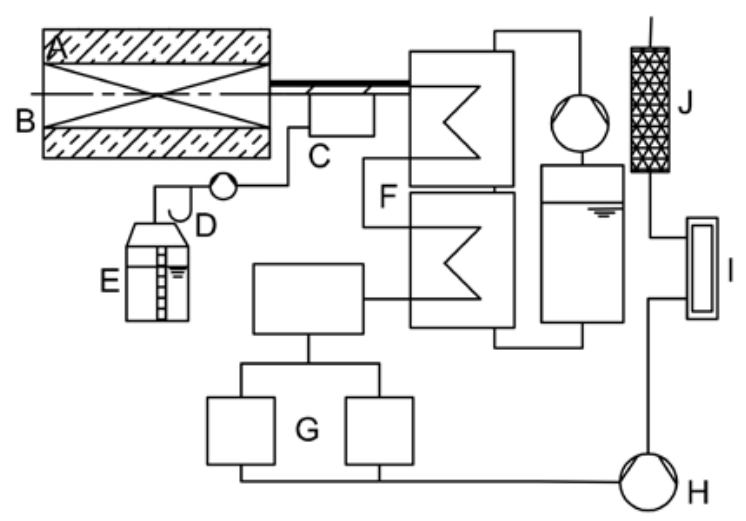

(a)
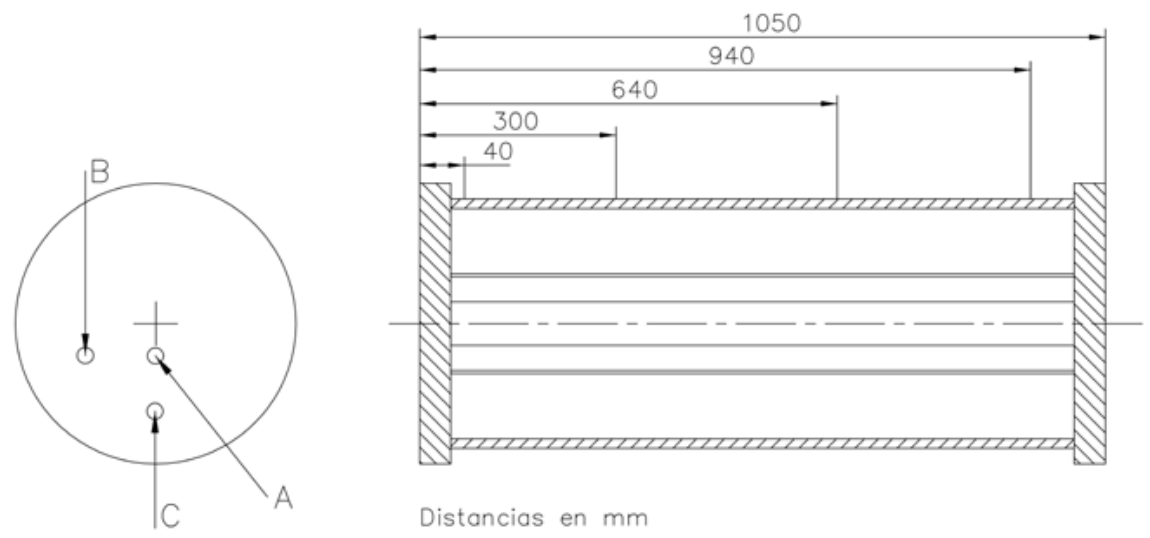

Distancias en $\mathrm{mm}$

(b)

Figura 3. (a) Montaje del reactor de lecho fijo horizontal para pirólisis biomasa. A. Sistema de alimentación. B. Reactor cilíndrico. C. Depósito de agua hielo con frascos para condensar alquitranes. D. Tubo en U. E. Depósito con agua a nivel graduado. F. Intercambiadores de calor. G. Filtros de aserrín para limpieza de gases. H. Bomba de vacío. I. Contador de gas. J. Chimenea. (b) Ubicación radial de termoelementos en el reactor horizontal.

Fuente: elaboración propia.

EN 14774-3, 14775 y 15148, respectivamente. Además, se determina la densidad granel según la norma DIN 51705 y el poder calorífico superior usando la correlación de Demirba (1997) que se presenta en la ecuación (1) y la correlación de Sheng y Azevedo (2005) que se presenta en la ecuación (2).

$$
\mathrm{HHV}=0.312 \mathrm{FC}+0.1534 \mathrm{VM}
$$

$$
\mathrm{HHV}=-3.0368+0.2218 \mathrm{VM}+0.2601 \mathrm{FC}
$$

HHV corresponde al poder calorífico superior, VM al material volátil y FC al carbono fijo
- Producto líquido: Al producto líquido se le determina el contenido de agua empleando el montaje descrito en la norma DIN 12420. El procedimiento para determinar el contenido de agua en el producto líquido, descrito en la norma, consiste en la codestilación con xileno, mezclando $100 \mathrm{ml}$ de xileno al 98,5 \% (blanco previamente calibrado), con una alícuota de alquitrán de 10 $\mathrm{ml}$. Luego se calienta durante $40 \mathrm{~min}$ a $130{ }^{\circ} \mathrm{C}$ y durante $15 \mathrm{~min}$ a $145^{\circ} \mathrm{C}$ (hasta cuando no se observan gotas de agua en el montaje excepto en la bureta recolectora de condensado), posteriormente se suspende el calentamiento, se deja enfriar a temperatura ambiente y se lee el nivel de 
la interfase agua/xileno condensado en la trampa de vapor o bureta.

- Producto gaseoso: Para las pruebas realizadas en la retorta (pruebas 1 y 3) y en el reactor de lecho fijo horizontal (prueba 4) se determina la composición del gas sometiéndolo a cromatografía de gases en un cromatógrafo marca Shimadzu modelo GC2010plus, cuyo principio de operación radica en la diferencia de conductividad térmica, tamaño molecular y punto de ignición detectada entre los compuestos. La composición permite además la estimación de propiedades físicas como densidad y fisicoquímicas como poder calorífico, mediante el cálculo de la propiedad por ley de mezclas. La ley de mezclas empleada consiste en la ponderación de las propiedades individuales de los compuestos con su respectiva composición. Ya que el montaje del reactor de lecho fijo vertical no permite la toma de muestra de gas, no fue posible tener esta información para la prueba 3. Para esta, sin embargo, es posible tener información sobre la producción instantánea de gas la cual se compara con la obtenida en la correspondiente prueba de la retorta.

\section{Manejo de información experimental}

Al final de cada prueba se determina la masa del carbonizado producido y el producto líquido, y se estima la cantidad de gas producido mediante un balance de materia. La masa de carbonizado remanente en el reactor, corresponde al que se extrae manualmente junto con el que se adhiere a las paredes y se retira con aire comprimido y herramienta abrasiva. La masa de producto líquido (agua y alquitrán generado por la pirólisis y agua condensada de la humedad del material), se determina sumando el líquido extraído de los recipientes de condensación y el calculado mediante el cambio de masa de los ductos o mangueras frías posteriores al reactor. Los rendimientos básicos se determinan con respecto a la masa inicial en base seca.

\section{RESULTADOS}

\section{Comportamiento térmico}

Los perfiles de temperatura de las diferentes pruebas se observan en la figura 4. Los perfiles de temperatura indican que fue posible reproducir

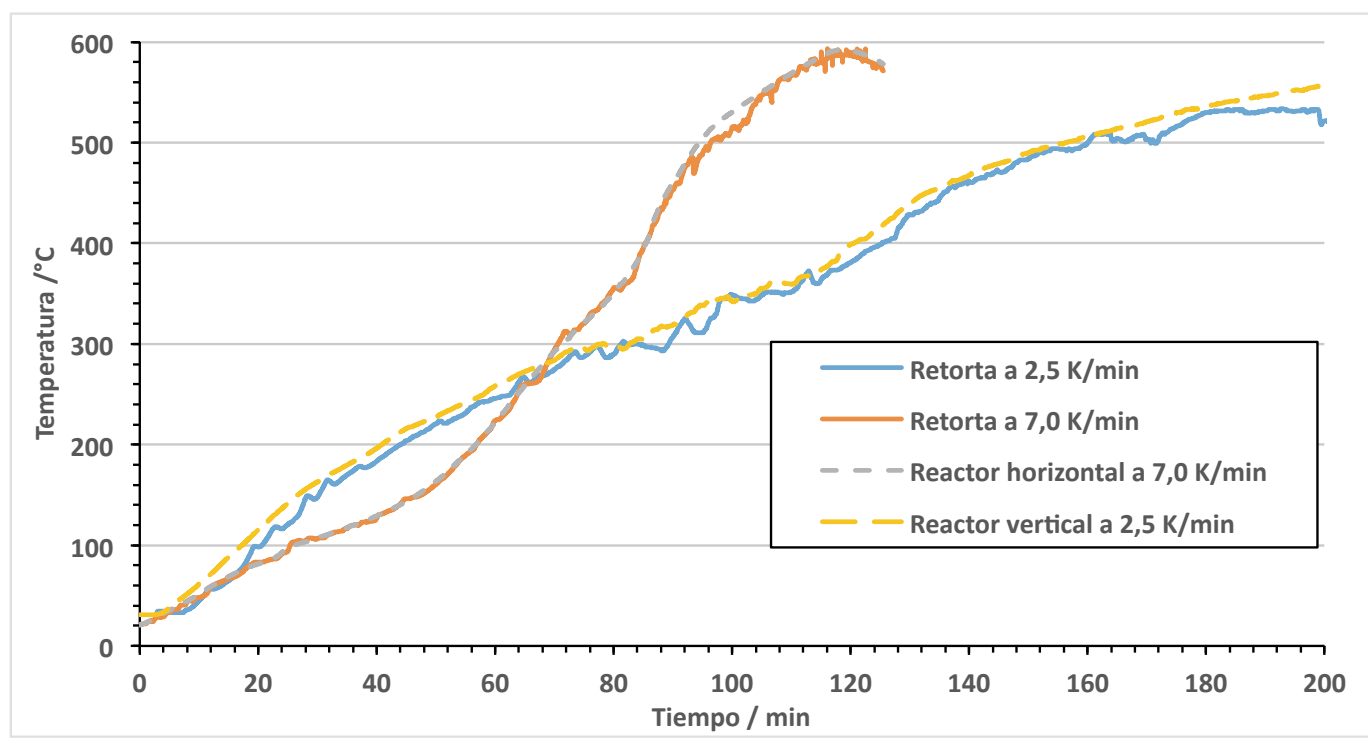

Figura 4. Perfiles de temperatura de las cuatro pruebas desarrolladas

Fuente: elaboración propia. 
el perfil térmico de los reactores en la retorta de manera satisfactoria. A partir de $150{ }^{\circ} \mathrm{C}$ se observa una tasa de calentamiento aproximadamente constante para los dos reactores, por lo que para cada perfil se calcula mediante regresión lineal a partir de esta temperatura. La tasas calculadas corresponden a $2,5 \mathrm{~K} / \mathrm{min}$ y $7,0 \mathrm{~K} / \mathrm{min}$.

\section{Rendimiento másico de la carbonización en las diferentes pruebas}

Los resultados de los rendimientos másicos para las diferentes pruebas se presentan en la figura 5 (a) para los experimentos realizados a $7 \mathrm{~K} / \mathrm{min}$ y en la figura 5 (b) para los experimentos realizados a $2,5 \mathrm{~K} / \mathrm{min}$. En las figuras se puede observar la variación en los rendimientos másicos con el tamaño de la muestra inicial utilizado.

En la figura 5 (a) se observa una alta influencia de las reacciones secundarias al escalar el proceso a nivel de la retorta de $0,16 \mathrm{I}$ al nivel de reactor de lecho fijo horizontal de 38,20 I. También se observa un aumento en la producción de carbonizado y de gas con un incremento en la masa inicial que se atribuyen a reacciones de recondensación de alquitranes y de craqueo respectivamente. Estas reacciones ocasionan que la fase líquida compuesta por alquitranes y agua disminuya. Los gradientes de temperatura en el lecho ocasionan que la biomasa reaccione inicialmente en la superficie y de manera progresiva en el interior de las partículas, a medida que incrementa la temperatura. De esta forma la fracción volátil proveniente del interior de la partícula cruza una capa de material ya carbonizado de la misma o de otra partícula y con una temperatura mayor, lo que promueve las reacciones secundarias. Las reacciones de craqueo se llevan a cabo principalmente por medio de craqueo térmico, reformado de vapor y reformado en seco de acuerdo con las ecuaciones (3), (4) y (5) (El-Rub, 2008). La disminución en la fracción de agua se puede deber a que la reacción de reformado de vapor, representada en la ecuación (4), se favorece en el proceso.

$$
\begin{gathered}
\mathrm{C}_{\mathrm{n}} \mathrm{H}_{\mathrm{x}} \rightarrow \mathrm{qC}_{\mathrm{m}} \mathrm{H}_{\mathrm{y}}+\mathrm{rH}_{2} \\
\mathrm{C}_{\mathrm{n}} \mathrm{H}_{\mathrm{x}}+\mathrm{nH}_{2} \mathrm{O} \rightarrow(\mathrm{n}+\mathrm{x} / 2) \mathrm{H}_{2}+\mathrm{nCO} \\
\mathrm{C}_{\mathrm{n}} \mathrm{H}_{\mathrm{x}}+\mathrm{nCO}_{2} \rightarrow(\mathrm{x} / 2) \mathrm{H}_{2}+2 \mathrm{nCO}
\end{gathered}
$$

En la figura 5 (b), donde se comparan las fracciones másicas en la retorta y en el reactor de lecho fijo vertical, no se obtienen cambios apreciables en las fracciones de carbonizado y gas. Este comportamiento indica que la influencia de reacciones secundarias de formación de carbonizado y craqueo de alquitranes en el reactor de lecho fijo vertical es menor que en el reactor horizontal. Esto se explica por el hecho de que en el reactor de lecho fijo vertical el gas de arrastre atraviesa el lecho causando una evacuación rápida de los productos volátiles obtenidos mientras que en el reactor de lecho fijo horizontal el gas de arrastre pasa por el espacio libre del reactor en la parte superior del lecho evitando que no haya una evacuación efectiva de los productos. En la misma figura se puede observar que aunque la fracción total de productos líquidos (alquitrán + agua) permanece constante, la fracción de alquitranes es mayor para el reactor de lecho fijo vertical que para la retorta. Estas variaciones pueden deberse a una alta influencia en la relación alquitrán/agua con la tasa de calentamiento durante la pirólisis. La parte más caliente del lecho alcanza una temperatura de $500{ }^{\circ} \mathrm{C}$ en 120 min mientras que la parte más fría en 210 min. Esta diferencia ocasiona una descomposición rápida en la parte inferior del lecho que favorece la producción de alquitranes obteniéndose al final del experimento una cantidad mayor de alquitranes que para el caso de la retorta. Sin embargo, este efecto debe ser estudiado en mayor profundidad en futuras investigaciones.

La influencia de la tasa de calentamiento en la fracción final de productos puede observarse al comparar los resultados de las pruebas 1 y 3 en las figuras 5 (a) y 5 (b) donde la producción de 


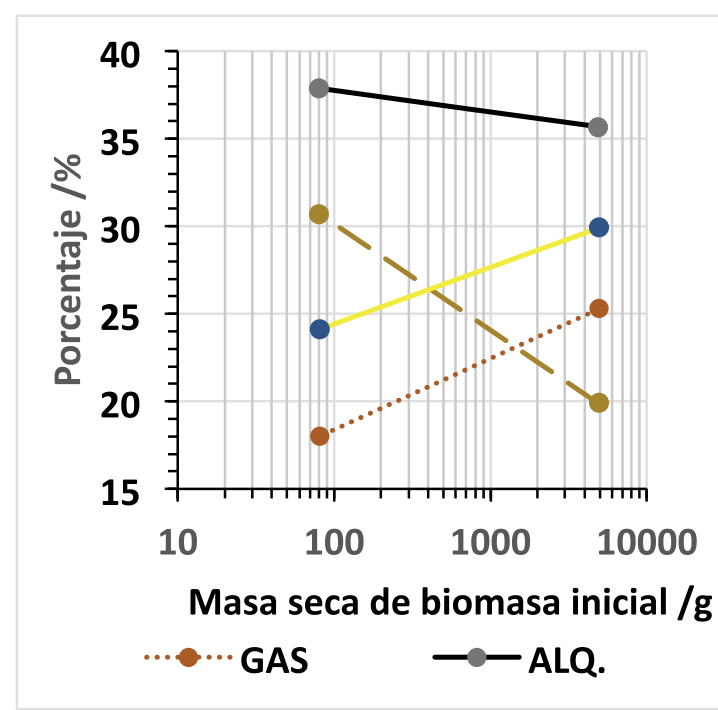

(a)

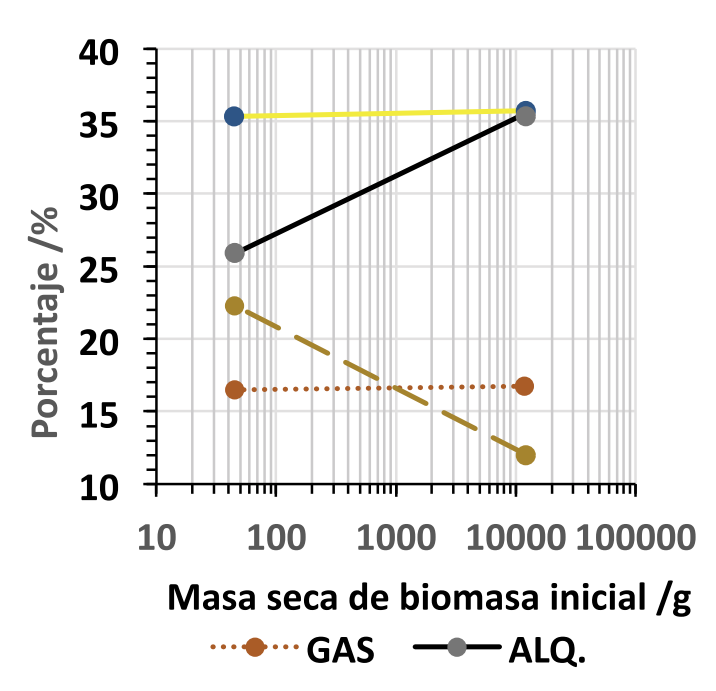

(b)

Figura 5. Variación del rendimiento másico de los productos con la masa seca de biomasa inicial. (a) Pruebas 3 y 4 realizadas a 7,0 K/min. (b) Pruebas 1 y 2 realizadas a $2,5 \mathrm{~K} / \mathrm{min}$

Fuente: elaboración propia.

alquitranes varía de $26 \%$ a $2,5 \mathrm{~K} / \mathrm{min}$ a $38 \%$ a $7 \mathrm{~K} / \mathrm{min}$.

Los resultados de las pruebas realizadas a $2,5 \mathrm{~K} / \mathrm{min}$ para la retorta son muy próximos a los reportados en la literatura a $3 \mathrm{~K} / \mathrm{min}$ por Gómez, Rincón y Klose (2010) y Suárez (2007) y los obtenidos para carbonización lenta por Kong, Loh y Bachmann (2014). El porcentaje de agua en los productos líquidos es para las pruebas con retorta a $2,5 \mathrm{~K} / \min$ y $7 \mathrm{~K} / \mathrm{min} 46 \%$ y $44 \%$ respectivamente. Para los productos líquidos obtenidos de los reactores de lecho fijo, el contenido de agua es de $36 \%$ para el reactor de lecho fijo horizontal y $25 \%$ para el reactor de lecho fijo vertical. Los valores reportados en literatura son muy variados ya que como se observa las fracciones dependen en gran medida de las condiciones utilizadas. Abnisa, Arami-Niya, Wan Daud, Sahu y Noor (2013) obtienen para racimo de aceite de palma un porcentaje de agua de los bioaceites producidos por pirólisis a $10 \mathrm{~K} / \mathrm{min}$ hasta $500{ }^{\circ} \mathrm{C}$ de 59 \%; Gómez et al. (2008a) reportan un valor de $50 \%$ para el porcentaje de agua en la fracción líquida para carbonización en retorta con una tasa aproximada de $6,5 \mathrm{~K} / \mathrm{min}$.

\section{Producción específica de gases}

En la figura 6 se representa la producción específica de gas acumulada y absoluta para las pruebas 1 y 2 , realizadas a la misma tasa de velocidad en la retorta y en el reactor de lecho fijo vertical.

La producción de gas es para las dos pruebas muy similar, esto concuerda con las observaciones de la sección anterior que indican una baja influencia de las reacciones secundarias para el reactor de lecho fijo. La producción de gas es mínima antes de $250^{\circ} \mathrm{C}$. Después de esta temperatura la biomasa comienza a degradarse rápidamente y produce los mayores flujos de gas en la operación alrededor de $300{ }^{\circ} \mathrm{C}$, la producción disminuye entre $350{ }^{\circ} \mathrm{C}$ y $400{ }^{\circ} \mathrm{C}$ seguida por un pequeño aumento hasta la temperatura final de procesamiento. Estos resultados concuerdan con los que obtuvieron Gómez et al. (2008b), quienes afirman que a partir de las curvas de variación de la masa de la fase sólida se reconocen de manera clara dos picos: uno alrededor de $260{ }^{\circ} \mathrm{C}$ y otro entre 335 y $365{ }^{\circ} \mathrm{C}$. Los picos se producen principalmente por la producción de $\mathrm{CO}$ y $\mathrm{CO}_{2}$. La producción de 


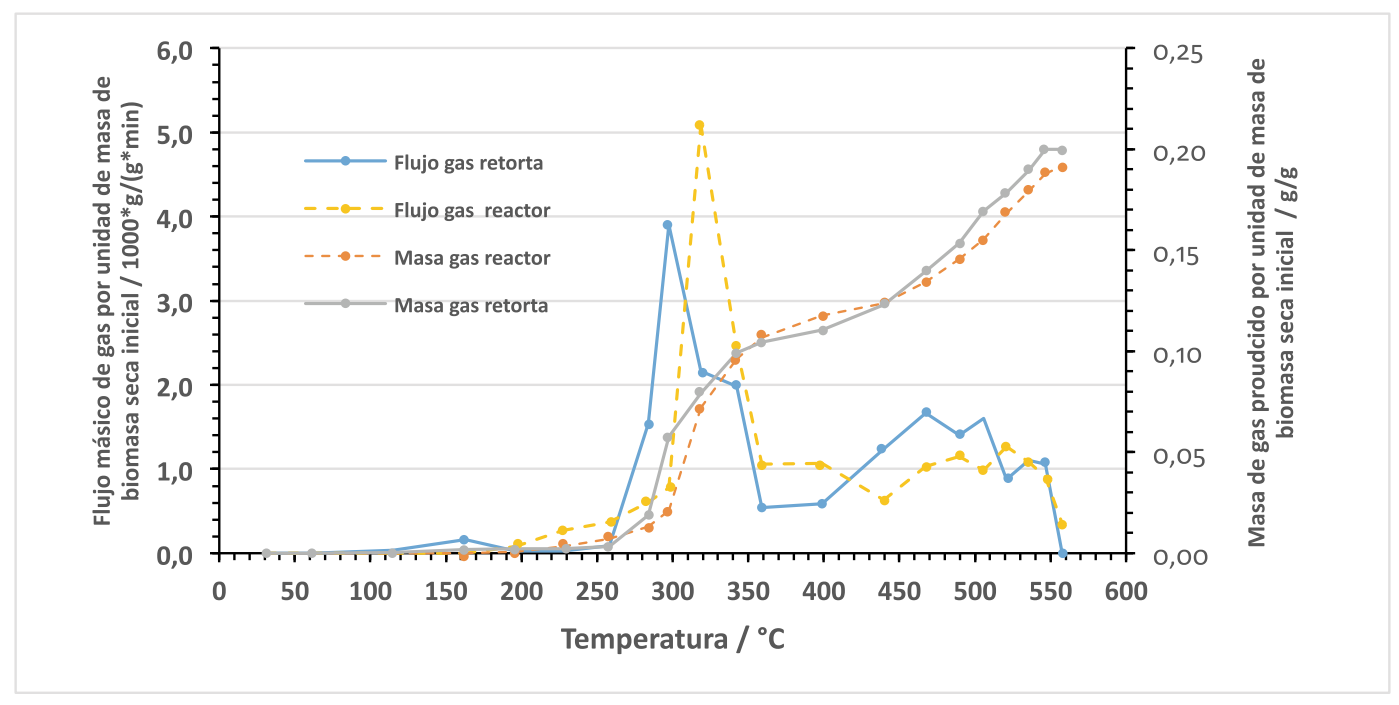

Figura 6. Producción de gases durante las pruebas 1 y 2 (retorta y reactor a 2,5 K/min)

Fuente: elaboración propia.

$\mathrm{CH}_{4}$ es significativa entre 400 y $500{ }^{\circ} \mathrm{C}$, mientras que la producción de $\mathrm{H}_{2}$ lo es a $700{ }^{\circ} \mathrm{C}$. Conesa y Domene (2011) afirman que la ubicación de los picos de producción de gases con respecto a la temperatura está asociada al tipo de componente de la biomasa. Los picos pronunciados entre 250 y $300{ }^{\circ} \mathrm{C}$ se asocian a contenido de hemicelulosa, entre 300 y $400{ }^{\circ} \mathrm{C}$ al contenido de celulosa, y entre 300 y $500{ }^{\circ} \mathrm{C}$ al contenido de lignina. Milhé, Van de Steene y Haube (2013) indican en cambio que este efecto se debe a la disposición de la estructura cristalina y amorfa del carbonizado, que cambia constantemente con la temperatura.

\section{Composición del gas}

Las muestras de gas tomadas del depósito de almacenamiento de gases para las pruebas 1, 3 y 4 se someten a cromatografía de gases para conocer la composición y determinar la densidad por ley de mezclas. En la figura 7 (a) se presentan los resultados para las dos pruebas realizadas en la retorta (pruebas 1 y 3) a 2,5 K/min y $7 \mathrm{~K} / \mathrm{min}$. En la figura 7 (b) se presenta la variación en la composición para las pruebas 3 y 4 realizadas a una tasa de calentamiento de $7 \mathrm{~K} / \mathrm{min}$ utilizando diferentes cantidades iniciales de biomasa. Las composiciones obtenidas concuerdan con valores reportados en literatura. Gómez et al. (2008a) reportan una composición de 28 vol. \% CO, 59 vol. \% $\mathrm{CO}_{2}$, 9 vol. \% $\mathrm{CH}_{4}$ y 4 vol. \% $\mathrm{H}_{2}$ para el gas de pirólisis de cuesco de palma de aceite en pruebas de termogravimetría a $10 \mathrm{~K} / \mathrm{min}$. En la figura 7 (a) se observa que a mayores tasas de calentamiento se fomenta la producción de $\mathrm{CO}_{2}$ y se obtienen menores concentraciones de $\mathrm{CH}_{4} \mathrm{e} \mathrm{H}_{2}$. La figura 7 (b) indica que a mayores tamaños de lecho se obtienen mayores concentraciones de $\mathrm{CH}_{4}$ e $\mathrm{H}_{2}$ y menores de $\mathrm{CO}_{2}$. La concentración de $\mathrm{CO}$ no varía apreciablemente en el desarrollo experimental empleado. A partir de la composición se calcula la densidad de los gases. Para el gas producido en la prueba con la retorta a $2,5 \mathrm{~K} / \mathrm{min}$ la densidad es de $1,42 \mathrm{~kg} / \mathrm{m}^{3}$, mientras que para el producido a $7,0 \mathrm{~K} / \mathrm{min}$ es de $1,58 \mathrm{~kg} / \mathrm{m}^{3}$. El poder calorífico del gas varía entre $6,14 \mathrm{MJ} / \mathrm{kg}$ para el gas producido en la prueba con la retorta a $7,0 \mathrm{~K} / \mathrm{min}$ y $22,62 \mathrm{MJ} / \mathrm{kg}$ para el gas producido en la prueba con el reactor horizontal a $7,0 \mathrm{~K} / \mathrm{min}$. Para la prueba en la retorta a $2,5 \mathrm{~K} / \mathrm{min}$ se obtiene un gas con un poder calorífico de 15,27 MJ/kg. 


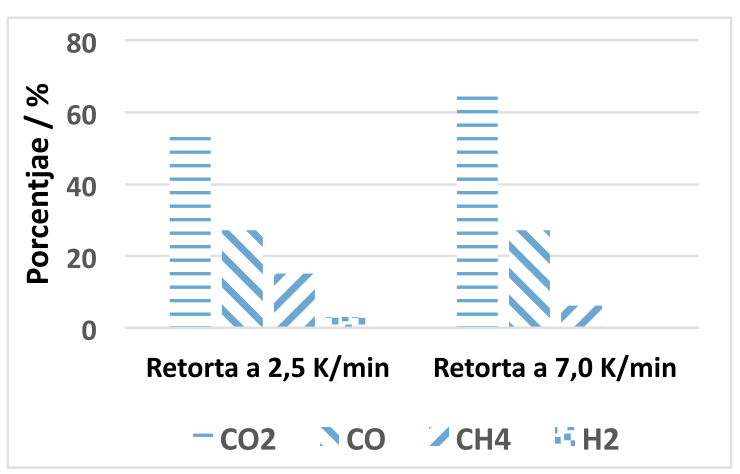

(a)

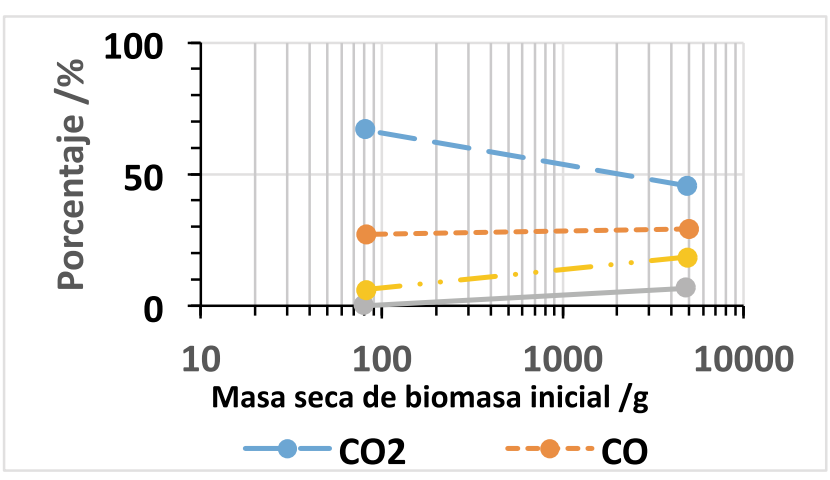

(b)

Figura 7. (a) Variación de la composición del gas producido con la tasa de calentamiento evaluada en la retorta para las pruebas 1 y 3. (b) Variación de la composición con la masa seca de biomasa inicial a 7,0 K/min

Fuente: elaboración propia.

\section{Caracterización de productos sólidos}

Los resultados del análisis próximo en base seca (humedad, contenido de volátiles y ceniza) se muestran en la figura 8. Para la materia prima se obtiene un contenido de humedad de $9 \%$, de materia volátil de $77 \%$, de ceniza de $2 \%$ y de carbono fijo (calculado por diferencia) de $12 \%$. A las condiciones dadas el orden de magnitud del porcentaje de volátiles y el de carbono fijo se intercambian cuando la biomasa se transforma en carbonizado. En la figura 8 se observa una mayor influencia en la variación del contenido de materia volátil y el carbono fijo para las pruebas realizadas en el reactor de lecho fijo horizontal que para el reactor de lecho fijo vertical, lo cual se explica por la menor influencia de la ocurrencia de reacciones secundarias en el segundo reactor, como se explicó en la sección anterior.

La densidad a granel de los carbonizados varía entre $555,3 \mathrm{~kg} / \mathrm{m}^{3}$ y $558,9 \mathrm{~kg} / \mathrm{m}^{3}$ y el poder calorífico superior entre $26,6 \mathrm{MJ} / \mathrm{kg}$ y $28,1 \mathrm{MJ} / \mathrm{kg}$.

El carbonizado disminuye la densidad en un $4,6 \%$, y aumenta su poder calorífico en promedio

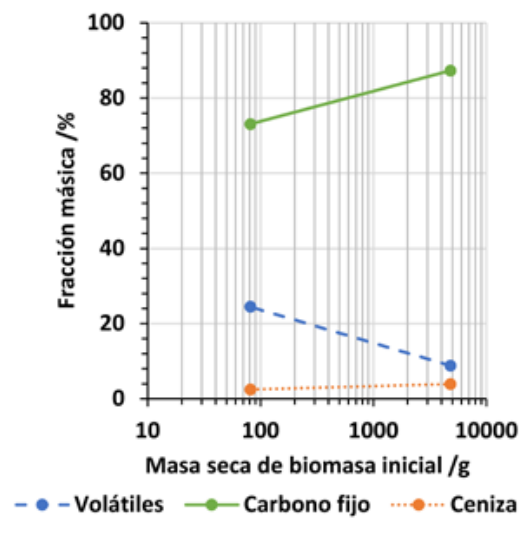

(a)

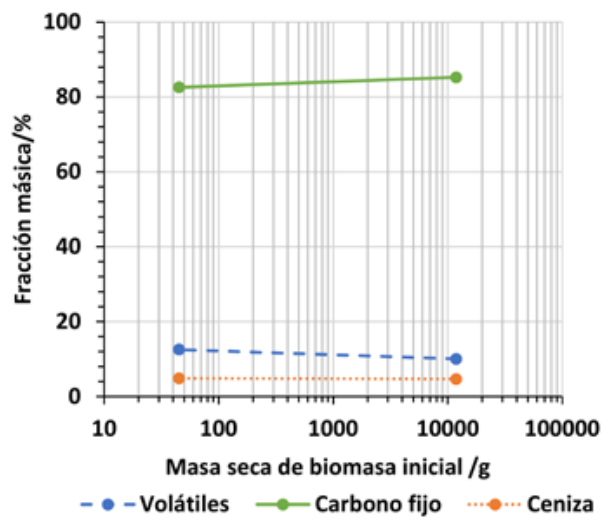

(b)

Figura 8. Variación del análisis próximo con la masa seca de biomasa inicial. (a) Pruebas 3 (retorta) y 4 (lecho fijo horizontal) a 7,0 K/min. (b) Pruebas 1 (retorta) y 2 (lecho fijo vertical) a 2,5 K/min

Fuente: elaboración propia. 
en un $27,4 \%$ con respecto a la materia prima inicial, que hacen del carbonizado un subproducto con buenas propiedades como combustible sólido.

\section{CONCLUSIONES}

Se determina una alta influencia del tamaño del reactor y la tasa de calentamiento en los rendimientos másicos y las características fisicoquímicas de los productos de la carbonización.

Al aumentar el tamaño del reactor aumenta la influencia de las reacciones secundarias de recondensación y craqueo de alquitranes que causan un aumento en el rendimiento de carbonizado y de gas, con una consecuente disminución del rendimiento de los productos líquidos. Adicionalmente, el craqueo de alquitranes provoca que parte de la energía de estos se transfiera al gas y, por tanto, que el poder calorífico del gas aumente. El poder calorífico del carbonizado no varía apreciablemente al modificar las condiciones del reactor. Al aumentar la tasa de calentamiento se favorece el rompimiento de cadenas en la biomasa que ocasiona el aumento en la producción de gases y líquidos. Por consiguiente, disminuye la producción de carbonizado.

El tipo de reactor y las condiciones de operación más conveniente para un producto energético depende de las características deseadas para el mismo. En el proceso de carbonización se desea producir la mayor cantidad de carbonizado, esto se logra utilizando bajas tasas de calentamiento y reactores de mayores tamaños de lecho de tal manera que se maximice la ocurrencia de reacciones secundarias de recondensación.

\section{FINANCIAMIENTO}

La Universidad Nacional de Colombia financió el desarrollo del proyecto "Obtención de gas de síntesis a partir de gasificación de cuesco de palma de aceite", código Hermes 19133.

El programa Jóvenes Investigadores e Innovadores, de Colciencias, financió al ingeniero David
Flórez a través de la convocatoria No. 617 de 2013.

\section{REFERENCIAS BIBLIOGRÁFICAS}

Abnisa, F.; Arami-Niya, A.; Wan Daud, W.; Sahu, J. y Noor, I. (2013). Utilization of oil palm tree residues to produce bio-oil and bio-char via pyrolysis. Energy Conversion and Managment, 76, 1073-1082.

Abnisa, F.; Wan Daud, W. y Sahu, J. (2011). Utilization possibilities of palm shells as a source of biomass energy in Malasya by producing bio-oil in pyrolysis process. Biomass \& Bioenergy, 35(5), 1863-1872.

Asadullah, M.; Ab Rasid, N.; Sharifah, A. y Azdarpour, A. (2013). Production and detailed characterizaton of bio-oil from fast pyrolysis of palm kernel shell. Biomass \& Bioenergy, 59, 316-324.

Basu, P. (2010). Biomass Gasification and Pyrolysis: Practical Design and Theory. Oxford, UK: Elsevier.

Campero R., O. (2010). La biomasa y los combustibles. Recuperado de: http://www.tecnologiasendesarroIlo.org/dw/11BIOMASA_1ERA_PARTE.pdf

Chen , W.-H. y Lin, B.-J. (2016). Characteristics of products from the pyrolysis of oil palm fiber and its peIlets in nitrogen and carbon dioxide atmospheres. Energy, 94, 569-578.

Conesa, J. y Domene, A. (2011). Biomasses pyrolysis and combustion kinetics through $\mathrm{n}$-th order parallel reactions. Thermochimica Acta,, 523(1-2) 176-181.

Demirba, A. (1997). Calculation of higher heating values of biomass fuels. Fuel, 76(5), 431-434.

El-Rub, Z. (2008). Biomass- char as an insitu-catalyst for a tar removal in gasification systems. Enschede, Holanda: Twente University.

Escalante H., H.; Orduz P., J.; Zapata L., H.; Cardona R., M. y Duarte O., M. (2008). Atlas del potencial energético de la biomasa residual en Colombia. Bogotá: UPME, Ideam, Colciencias, Universidad Industrial de Santander. Recuperado de: http://www1.upme. gov.co/sites/default/files/article/1768/files/Atlas\%20 de\%20Biomasa\%20Residual\%20Colombia_.pdf

Faaij, A. (2006). Modern biomass conversion technologies. Mitigation and Adaptation Strategies for Global Change, 11, 343-375. 
Fedepalma (2013). Síntesis del proyecto GEF, Conservación de la biodiversidad en las zonas de Cultivos de Palma. Bogotá. Recuperado de: http://web. fedepalma.org/sites/default/files/files/Fedepalma/2013-05_S\%C3\%ADntesisProyectoGEF.pd

Firdaus Mazlan, M.A.; Uemura , Y., Osman , N., \& Yuzup , S. (2015). Fast pyrolysis of hardwood residues using fixed bed drop-type pyrolyser . Energy Conversion and Management, 98, 208 - 2014

Gómez, A.; Klose, W. y Rincón, S. (2008a). Pirólisis de Biomasa, Cuesco de Palma. Kassel, Alemania: Kassel University Press.

Gómez, A.; Klose, W. y Rincón, S. (2008b). Thermogravimetrische Untersuchungen zur Pyrolyse von Ölpalmschalen. Chemie Ingenieur Technik, 80, 1-11.

Gómez, A.; Rincón, S. y Klose, W. (2010). Carbón activado de cuesco de palma. Kassel, Alemania: Kassel University Press.

González H., A.; García U., C. y Talero R., G. (2014). Estudio de planta piloto para peletización de residuos madereros y su utilización como combustible en hornos ladrilleros. Tecnura, 18(40), 62-70.

International Energy Agency (IEA) (2011a). Total primary energy supply Colombia. Recuperado de: http://www.iea.org/statistics/

International Energy Agency (IEA) (2011b). Total primary energy supply world. Recuperado de: http:// www.iea.org/statistics

Kong, S.H.; Loh, S.K. y Bachmann, R. (2014). Biochar from oil palm biomass: A review of its potential and challenges. Renewable and Sustainable Energy Reviews, 39, 729-739.

McKendry, P. (2001). Energy Production from Biomass (Part 2): Conversion Technologies. Biosource Technology, 83, 47-54.

McKendry, P. (2002). Energy production from biomass (Part 1): Overview of Biomass. Bioresource Technologie, 83, 37-46.

Milhé, M.; Van de Steene, L. y Haube, M. (2013). Autothermal and allothermal pyrolysis in a continuous fixed bed reactor. Journal of Analytical and Applied Pyrolysis, 103, 102-111.

Ninduangdee, P.; Kuprianov, V.; Young Cha, E. Y Kaewrath, R. (2015). Thermogravimetric studies of oil palm empty fruit bunch and palm kernel shell: TG/DTG analysis and modelling,. Energy Procedia, 79, 453-458.

Nuñez, C. y Danny, W. (2012). Uso de residuos agrícolas para la producción de biocombustibles en el departamento del Meta. Tecnura, 16(34), 142-156.

Anaya-Durand, A. y Pedroza-Flores, H. (2008). Escalamiento, el arte de la ingeniería química: Plantas piloto, el paso entre el huevo y la gallina. Tecnología, Ciencia, Educación, 23(1), 31-39.

Senneca, O. (2007). Kinetics of pyrolysis, combustion and gasification of three biomass fuels. Fuel Processing Technology, 88, 87-97.

Seon-Jin, K.; Su-Hwa, J. y Joo-Sik, K. (2010). Fast pyrolysis of palm kernel shells: Influence of operation parameters on the bio-oil yield of phenol and phenolic compounds. Biosource Technology, 101, 9294-9300.

Sheng, C. y Azevedo, L. (2005). Estimating the higher heating value of biomass fuels from basic analysis data. Biomass and Bioenergy, 28, 499-507.

Suárez Romero, M. (2006). Investigación de la distribución de los productos de la biomasa según la norma ISO 647. Tesis de Pregrado. Bogotá: Universidad Nacional de Colombia.

Uemura, Y.; Saadon, S.; Osman, N. y Mansor, N. (2015). Torrefaction of oil palm kernel shell in the presence of oxygen and carbon dioxide. Fuel, 144, 171-179.

Ugur, M. y Sevgi, S. (2015). Pyrolysis of Hornbeam Shell (Carpinus betullus L.) in a fixed bed reactor: Characterization of bio-oil and bio char. Fuel, 150, 672-678.

Vassilev, S.; Baxter, D.; Andersen, L. y Vassileva, C. (2013). An overview of the composition and application of biomass ash. Part 1. Phase-mineral and chemical composition and classication. Fuel, 105, 40-76.

Zhongking, M.; Dengyu, C.; Gu, J.; Bao, B. y Qisheng, Z. (2015). Determination of pyrolysis characteristics and kinetics of palm kernel shells using TGAFTIR and model free integral methods. Energy Conversion and Management, 89, 251-259. 